\title{
Recent Development of Polymer Network Liquid Crystal Displays
}

\author{
Toru FUJISAWA, Hidetoshi NAKATA, Masao AIZAWA \\ Central Research Laboratories, Dainippon Ink and Chemicals, Inc. \\ 631, Sakado, Skura-shi, Chiba-ken, 285, Japan
}

\begin{abstract}
We have studied the electro-optical properties in Polymer Network Liquid Crystal Displays which are composed of a three-dimensional polymer network formed in continuous liquid crystal layers prepared by photo-polymerization induced phase separation processes. In view of the behavior in the orientation of the liquid crystal molecule at the polymer's surface, it can be clarified that the electro-optical properties are strongly governed by the polymer. By a combination of two different characteristics of the polymers with respect to a temperature dependence, a variation in the driving voltage for a temperature change can be improved.

Keywords: polymer network liquid crystal displays, anchoring transition, acrylate
\end{abstract}

\section{Introduction}

Polymer-dispersed liquid crystals (PDLC) [14], dispersed nematic droplets in a polymer matrix, have attracted much attentions to the various application such as reflective direct view displays and light valves for projectors, because of the considerable decrease in light loss compared with the commonly used Twisted Nematic-LCDs.

We have investigated Polymer Network Liquid Crystal Displays (PNLCD) as the light modulator possessing the capability of controlling the degree of light scattering using an applied electric field.

To prepare the PNLCD, photo-polymerization induced phase separation processes are used. The morphology of the films in the PNLCD are different from PDLC films in which a nematic droplet is perfectly surrounded by a polymer matrix and isolated from each other. The morphology of the PNLCD features a three-dimensional polymer network formed in continuous liquid crystal layers as shown in Figure 1. The liquid crystalline molecules in the PNLC films are randomly oriented to the surface of the polymer network so that the LC molecules create a multidomain. This LC molecular orientation causes a strong scattering effect on incident light. The light scattering effect is influenced by the birefringence of the LC mixture and by the mesh size of the polymer network. When an electric filed is applied across the $\mathrm{LC}$ cell, the LC molecules are aligned in the direction of the electric field, and the films become transparent with a transmittance higher than $85 \%$. Moreover, a lower driving voltage of less than $4 \mathrm{Vrms}$ can be achieved with high resistivity and high contrast by careful selection of the polymer using optimized curing conditions [5-7]. Such a PNLCD has been successfully applied to a CdSe TFT active matrix $[8,9]$. However, the subject concerning variation in the electro-optical properties for a temperature change is greater in practical applications, because the variation in the electro-optical properties such as the driving voltage on PDLC [10] or PNLC is greater than a conventional TN-LCD. In order to suppress the variation in the electro-optical properties for a temperature change, it is important that the mechanism for the electro-optical switching is investigated.

In this paper, we describe the electro-optical properties as a function of temperature in the PNLCD influenced by the anchoring transition. In addition, the method to control the temperature dependence of the driving voltage is proposed.

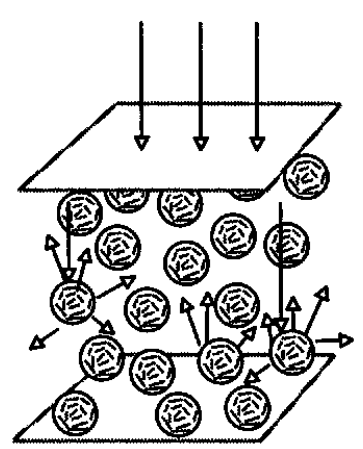

PDLC-LC droplets

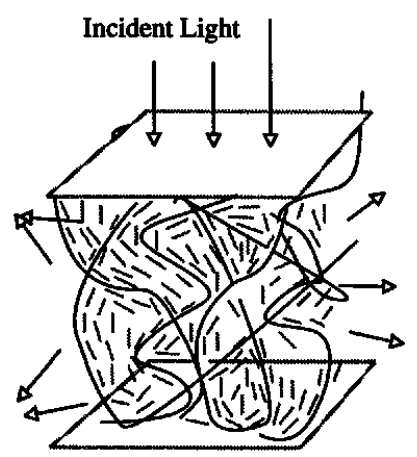

PNLC-Continuous LC layer
Fig. 1. The differences of morphology between PDLC and PNLC 


\section{Experimental}

\subsection{Sample Preparation}

Fluorinated liquid crystals and UV-curable acrylic monomers doped with $0.4 \%$ of the photoinitiator (Irgacure-651;Ciba Geigy) are used to prepare the PNLCD. The same liquid crystals which have a nematic phase in the temperature range 1$103^{\circ} \mathrm{C}$ are employed in all samples for this study. After filling the materials in an LC cell with a $11 \mu \mathrm{m}$ cell gap, $20 \mathrm{mw} / \mathrm{cm}^{2}$ of UV light is irradiated for 60 seconds to form the three-dimensional polymer network at a temperature above isotropic-nematic transition temperature.

\subsection{Measurement of Electro-optical Properties}

The voltage-transmittance properties are measured with a $1 \mathrm{kHz}$ sine waveform through a F\#4.5 optical system. V90 is defined as the voltage requiring $90 \%$ of the total transmittance change. To is defined as the minimum transmittance in the total change.

\subsection{Measurement of Anchoring Strength}

The high-electric-filed technique which eliminates the capacitance measurement at high AC voltage devised by Dr. H. Yokoyama $[10,11]$, was used to determine the extrapolation length for a temperature change in the hybrid LC cell combined with the homeotropic alignment layer and UV-curable acrylic alignment layer.

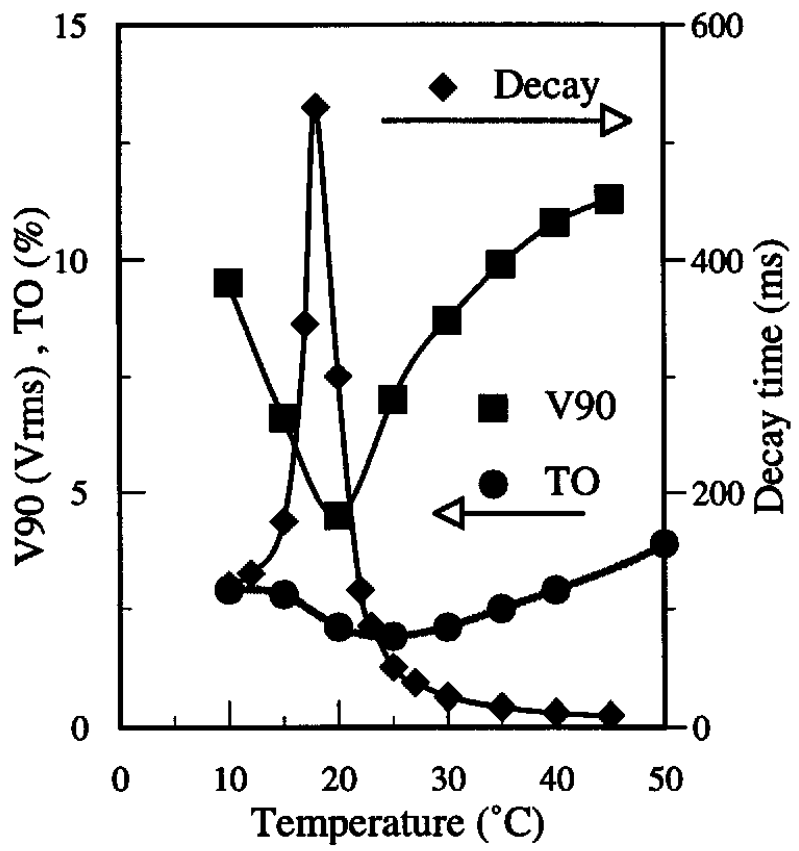

Fig. 2. A example of the opposite temperature dependence of electro-optical properties.

\section{Temperature dependence of electro-optical properties}

In a typical PNLCD, the V90 is a decrease due to increasing temperature. The variation in V90 for almost all polymers used in the PNLCD is in the range approximately 0.1 Vrms to over $0.3 \mathrm{Vrms}$ per $1^{\circ} \mathrm{C}$. However, in some compositions, the opposite temperature dependence of V90 in which V90 increased as the temperature increased is observed in a certain temperature range. Such an opposite temperature dependence of V90 as a function of temperature is shown in Figure 2. The minimum point of V90 can be seen at $20^{\circ} \mathrm{C}$. Furthermore, the inflection point in T0 for minimum transmittance as a function of temperature and the decay time peak on the switching time are observed in the vicinity of the same temperature indicating the minimum V90 dependence on temperature in Figure 2. In general, T0 gradually decreases as the temperature decreases due to increasing birefringence of the $\mathrm{LC}$ mixture. Nevertheless, T0 rapidly increased below $20^{\circ} \mathrm{C}$. For the decay time as a function of temperature, the decay time of 49 msec at $25^{\circ} \mathrm{C}$ jumped to $530 \mathrm{msec}$ at $18{ }^{\circ} \mathrm{C}$ with only a slight temperature change. It can be seen that these discontinuous changes in the electro-optical properties are not caused by the physical properties of liquid crystals such as elastic constant, birefringence, dielectric anisotropy and viscosity, because no such abrupt changes are observed in the vicinity of $20^{\circ} \mathrm{C}$. Therefore, it is considered that the interaction between the liquid crystals and polymers may significantly affect the factor that determines the temperature dependence of the electro-optical properties.

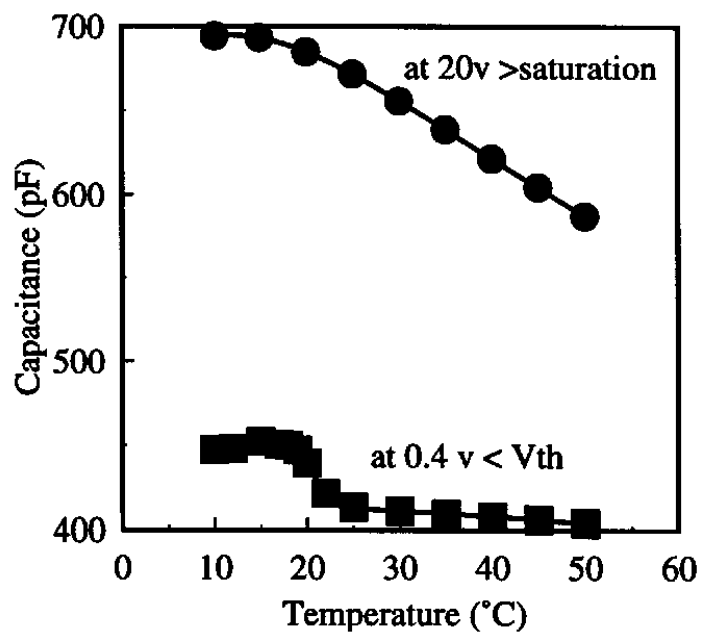

Fig. 3. Capacitance in off-state and in on-state as a function of temperature. 


\section{Anchoring transition}

It is presumed that the increase in $\mathrm{TO}$ below $20^{\circ} \mathrm{C}$ is attributed to the change in the director orientation at the surface of the polymer. In order to examine the internal behavior based on the reorientation of the director, capacitance as a function of temperature in the PNLCD is measured. Figure 3 shows the capacitance in the off-state applied below the threshold voltage and in the on-state applied over the saturation voltage as a function of temperature. The abrupt change in the capacitance curve at the off-state is observed below $20^{\circ} \mathrm{C}$. Such behavior is due to the change in the director, though increasing capacitance during the on-state with decreasing temperature is affected by the behavior of the dielectric anisotropy.

Furthermore, in order to clarify the reorientation of the director for a temperature change, polarizing microscopic observation was carried out with a PNLCD having a mesh size over $10 \mu \mathrm{m}$. The microscopic photograph for the different temperature is shown in Figure 4. In Figure 4 (a), the white line represents the homeotropic orientation neighbor of the vertical wall of polymer network surface normal to the substrate in the LC cell, and the black area represents the homeotropic orientation at the horizontal wall. Accompanying an increase in temperature, it can be seen that the director orientation is varied from a homeotropic orientation in the vicinity of $20^{\circ} \mathrm{C}$ as shown in Figure 4 (a) to a homogeneous orientation as shown in Figure 4 (b). Apparently, these phenomena are governed by the reorientation of the director at the polymer surface. Such reorientation behavior is on the basis of the anchoring transitions in which the change in the director of orientation at the polymer surface spontaneously occurs by a variation in temperature.

The anchoring transition was first reported in 1976 [11]. The reorientation of the director with the sequence tilted-homeotropic-tilted is occurred in the vicinity of the clearing point on the rubbed glass substrate covered with a thin amorphous carbon film for nematic MBBA. In the PNLCD, the anchoring transition temperature is observed even at a lower temperature range. This anchoring transition is depended on the kinds of the liquid crystal mixture and the UV-curable acrylic monomer composition. The anchoring transition temperature in which is defined as the temperature indicating the minimum value of the V90 as a function of temperature increases in proportion to the number of carbons in the alkyl chain of the alkyl acrylate, and as increasing the amount of the alkyl acrylate in the acrylic monomers. From the standpoint based on the influence of the alkyl acry- late including the molecular structure and the concentration of alkyl acrylate, the intermolecular forces and a density of the alkyl chain at a polymer surface are given as the factors for the anchoring transition.
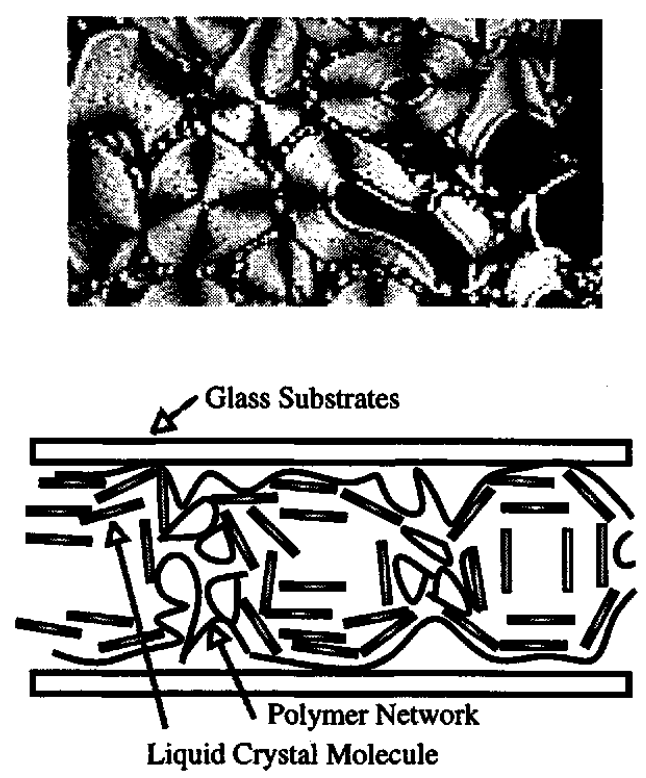

(a) Homeotropic structure at $20^{\circ} \mathrm{C}$
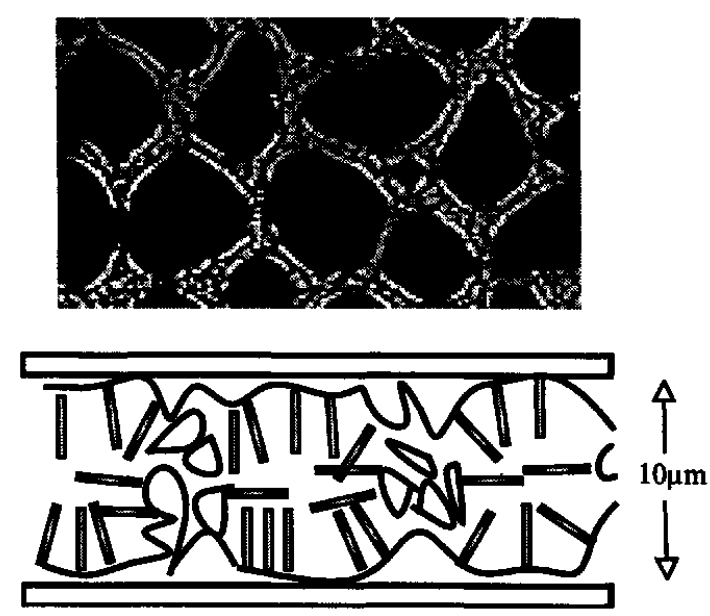

(b) Transition of homogeneous structure at $35^{\circ} \mathrm{C}$

Fig. 4. Polarizing microscopic photograph. 


\section{Extrapolation length}

The influence of intermolecular forces are considered from the variation in the V90 and the decay time for a temperature change caused by the anchoring transition. In order to evaluate the interaction between liquid crystalline molecule and polymer, the measurement of extrapolation length in the hybrid LC cell combined with a homeotropic alignment layer and the UV-curable acrylate alignment layer in which consists of the same monomer composition in the PNLCD is performed using the high electric field technique described above. An extrapolation length as a function of temperature in the case having $32^{\circ} \mathrm{C}$ anchoring transition temperature is shown in Figure 5. The extrapolation length is drastically increased in the vicinity of the anchoring transition temperature. The extrapolation length varied more than 4 times above the anchoring transition temperature within a few ${ }^{\circ} \mathrm{C}$ of the temperature. The extrapolation length decreased with an asymptotic curve with increasing temperature. The increase of extrapolation length implies a decrease of intermolecular forces. Therefore, it turned out that the abrupt changes in the electro-optical properties mentioned above are caused by the drastic change in the anchoring forces near the anchoring transition at the polymer's surface.

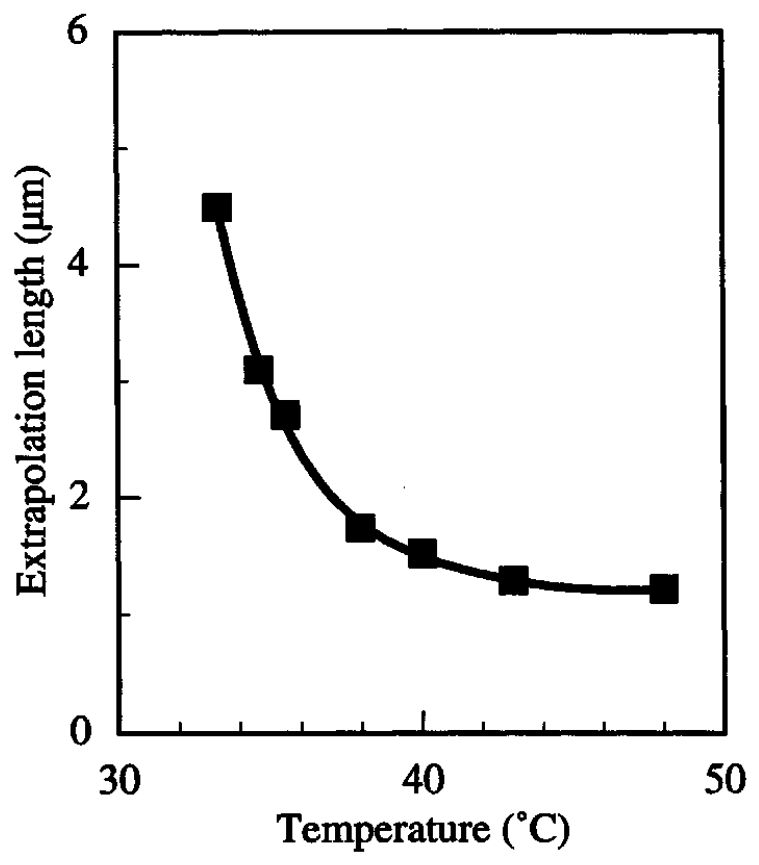

Fig. 5. Extrapolation length as function of temperature.

\section{Controlling the variation in V90 for a tempera- ture change}

We can successfully control V90 as a function of temperature by the combination of two different characteristics of the polymers with respect to temperature dependence. One characteristic of the polymers such as polymer $B$ having no anchoring transition noted in Figure 6 is that V90 is monotonously decreased with increasing temperature. The other characteristic of the polymers such as polymer A noted in Figure 6 is that V90 is influenced by the anchoring transition. In this case, the anchoring transition temperature is at $5^{\circ} \mathrm{C}$. When polymer $\mathrm{A}$ is combined with polymer $B$, the variation in $\mathrm{V} 90$ for a temperature change is suppressed as shown in Figure 6. As a result, the minimal variation in V90 within 0.5 Vrms as shown in Figure 7 can be attained in the region of $10^{\circ} \mathrm{C}$ to $60^{\circ} \mathrm{C}$ with an adequate amount of the monomer indicating the anchoring transition.

We can also realize the slight variation in V90 for a temperature change as shown in Figure 8 with the other means. In this case, the polymer indicating the anchoring transition is only used to control the variation of V90 for a temperature change. This means can be achieved by the adjusting the effect in the anchoring transition with the appropriate length in the alkyl chain and the adequate alkyl density on the polymer's surface.

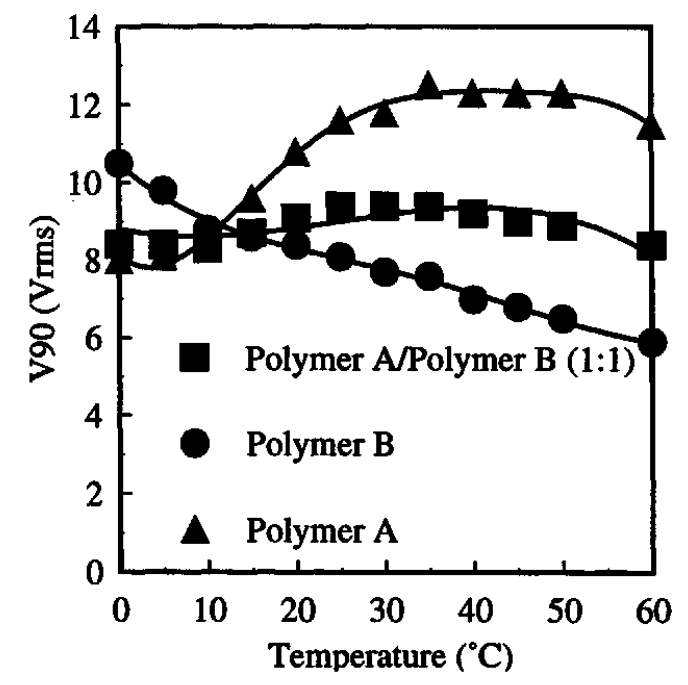

Fig. 6. Influence of polymers for a temperature change. 


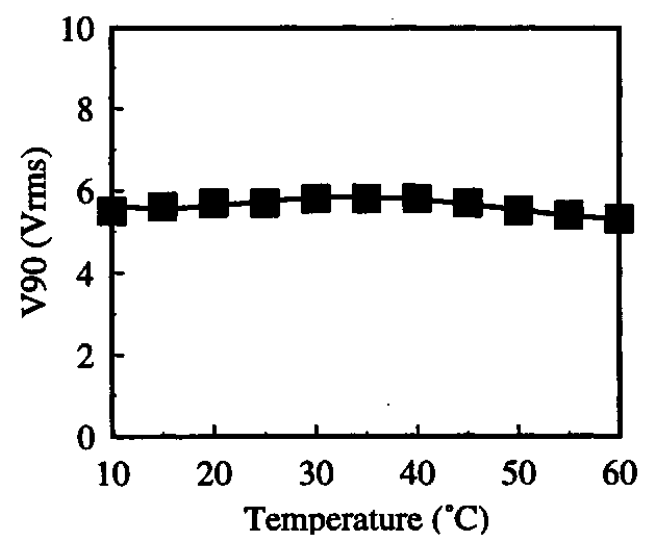

Fig. 7. The improvement of temperature dependence on V90 with the combination of two different characteristics of the plymers with repect to temperature dependence.

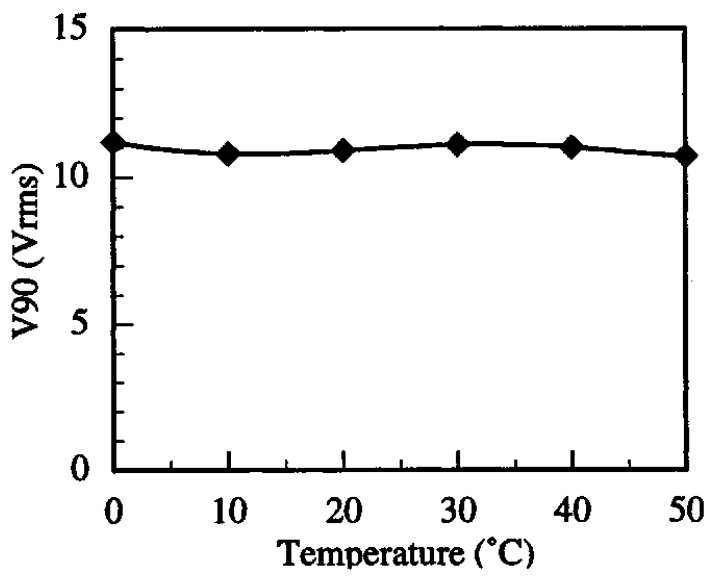

Fig. 8. The improvement of temperature dependence on V90 with only using the polymers indicating the anchoring transition.

\section{Conclusion}

It can be seen that the opposite temperature dependence in which V90 increased with increasing temperature in a certain temperature range is caused by the behavior due to the reorientation of the director near anchoring transition temperature. Moreover, it has become apparent that extrapolation length is significantly varied in the vicinity of the anchoring transition by the measurement using the high electric field technique. In other words, anchoring forces are weakened near the anchoring transitions. Consequently, from the consideration of the anchoring transitions, it can be proven that the electro-optical properties in the PNLCD strongly depends on the orientation at the polymer's surface. By utilizing the effect of anchoring transitions, the variation in V90 can be suppressed.

\section{Acknowledgments}

The authors are very grateful to $\mathrm{Dr}$. $\mathrm{H}$. Yokoyama and Dr. Sun for the useful discussions and measurement of the extrapolation length.

\section{References}

1. Fergason,J.L.,SID Dig.Tech. Paper,16 (1985) 68.

2. Drzaic,P.S., J.Appl.Phs.,60 (1986) 2142.

3. Doane,J,W.,Vaz,N.A., Mu,B.G., and Zummer,S., Appl. Phys, Lett.,48 (1986) 269.

4. Drzaic,P.S., Lqu.Cryst.,3 (1988) 1543.

5. Fujisawa,T.,et al,9thInt.DisplayResearch Conf.,690 (1989).

6. Fujisawa,T.,et al,13thInt.Liq.Cryst.Conf.,II (1990) p.21.

7. T. Fujisawa, et al, Asia Display`95, SID, (1995) p431-434.

8. J. De Beats, et al, Int. Display Research Conf. 91, SID (1991) p.215-218, (San-Diego).

9. J. De Beats, et al,Proc. of the 1st TFT symposium, (October 1992) p.296-306, The

Electrochem.Soc. (Toronto).

10. David Coates, J. Mater. Chem., 5(12),(1995) 2063-2072

11. Yu. Ryschenkov and M. Kleman, J. Chem.

Phys. 64 (1976) 404 